\title{
PRE-FILLED SYRINGE AS AN OPTION FOR DRUG DELIVERY IN EMERGENCY MEDICINE
}

\author{
Marcin Madziala ${ }^{1}$, Jacek Smereka², Lukasz Szarpak ${ }^{1}$ \\ ${ }^{1}$ Department of Emergency Medicine, Medical University of Warsaw, Warsaw, Poland \\ ${ }^{2}$ Department of Emergency Medical Service, Wroclaw Medical University, Wroclaw, Poland
}

Disaster Emerg Med J 2017; 2(2): 98-99

\section{Dear Editor,}

The advance preparation of intravenous drugs is a common practice in healthcare in order to deal with emergency situations. This practice also has other drawbacks such as the potential confusion of drugs, mistakes when reconstituting a drug, drug labelling errors or the risk of contamination. The most prevalent preparation and injection equipment in Poland are needles and syringes. Manufactured according to generally accepted principles and markings, they are sterile and always disposable. Any injection requires medical staff to follow the full procedure of preparation and administration. In medical emergency settings due to the multitude of other activities at the scene and the dynamics of the patient's health state changes, the personnel preparing the drug before the injection may commit a number of significant errors. When preparing a medication for administration, the personnel performing the procedure is exposed to injury when the drug ampoule is broken with a risk of a potential infection. Another problem is also the glass particles that may remain in the broken ampoule which can enter the bloodstream of the patient while aspirating the drug to the syringe. The next potential problem is the wrong selection of the pharmaceutical agent by confusing the ampoule, and it is often noted that the inscriptions on the ampoules are smeared so that the name and expiry date of the drug are no longer visible. The necessity of diluting IV drugs also is neglected, especially during cardiopulmonary resuscitation.

In order to prevent this situation the pre-filled syringes are widely used in western countries. Perhaps such pre-filled syringes will be available also for the Polish pharmaceutical market soon. Their advantages undoubtedly include the fact that the drug contained within is prepared by the manufacturer. The syringe is sterile and does not require any special preparation by the medical staff, while the dose is used as a standard treatment.

Due to the limited time for medical personnel during cardiopulmonary resuscitation, the dynamics of the rescue operation and the rapid changes in the patients' condition, the duration of drug preparation may be crucial in certain emergencies, including anaphylaxis or cardiac arrest settings. The reduced time of drug preparation can potentially reduce the stress for the paramedic involved while the drug can be delivered to the patient immediately.

It is very important that the patient not incur additional financial and health care costs. Despite the apparently higher costs of pre-filled syringes, in fact, pre-filled syringes can reduce the total costs as a result of reducing the exposure to the risk for the medical personnel and the number of complications that may occur at the time of preparation. The use of the prefilled syringe not only shortens the preparation procedure but also minimizes the adverse effects that result from non-compliance by medical personnel such as infection, drug, dose or route of administration errors.

Zhao et al. [1] suggest the occurrence of glass delamination is a serious concern for parenteral drug products, which is not the case for pre-filled syringes. Benhamou et al. [2] showed that even though atropine PFS are more expensive than atropine in ampoules, its use may lead to significant cost savings. The reduced costs are mainly related to 
a reduced number of medical errors and the elimination of wastage in connection with atropine syringes prepared in advance.

The use of pre-filled syringes also saves medical staff time as a result of the preparation procedure. The use of the pre-filled syringe is complete and the patient receives a precise dose ordered in a given emergency procedure and is not exposed to a lower dose as a result of losses during preparation. Adapa et al. [3], indicated that during simulated patient with septic shock, nurses took $156 \mathrm{~s}$ to start infusions when using pre-filled syringes compared with $276 \mathrm{~s}$ when preparing them de novo, a mean delay of $106 \mathrm{~s}(\mathrm{p}<0.001)$.

In conclusion, there are a number of review articles that describe the advantages of pre-filled syringes $[4,5]$. The routine use of pre-filled syringes containing resuscitation drugs should therefore be considered in pre-hospital emergency care as providing drug infusions in syringes pre-filled by pharmacists or pharmaceutical companies would reduce medication errors and treatment delays, and improve patient safety.
Conflict of interest: None declared.

\section{REFERENCES}

1. Zhao J, Lavalley V, Mangiagalli $P$, et al. Glass delamination: a comparison of the inner surface performance of vials and pre-filled syringes. AAPS PharmSciTech. 2014; 15(6): 1398-1409, doi: 10.1208/s12249014-0167-y, indexed in Pubmed: 24938618.

2. Benhamou D, Piriou V, De Vaumas $C$, et al. Ready-to-use pre-filled syringes of atropine for anaesthesia care in French hospitals - a budget impact analysis. Anaesth Crit Care Pain Med. 2017; 36(2): 115-121, doi: 10.1016/j.accpm.2016.03.009, indexed in Pubmed: 27481691.

3. Adapa RM, Mani V, Murray $\sqcup$, et al. Errors during the preparation of drug infusions: a randomized controlled trial. Br J Anaesth. 2012; 109(5): 729-734, doi: 10.1093/bja/aes257, indexed in Pubmed: 22850220.

4. Murdoch $\mathrm{H}$, Jordan L, Tuckey J. Pre-filled thiopental syringes reduce cost and wastage whilst improving safety. Int J Obstet Anesth. 2012; 21(4): 384-385, doi: 10.1016/j.ijoa.2012.06.004, indexed in Pubmed: 22918026.

5. Adapa RM, Mani V, Murray $U$, et al. Errors during the preparation of drug infusions: a randomized controlled trial. Br J Anaesth. 2012; 109(5): 729-734, doi: 10.1093/bja/aes257, indexed in Pubmed: 22850220. 